\title{
Photometry and models of selected main belt asteroids
}

\section{184 Dejopeja, 276 Adelheid, 556 Phyllis}

\author{
A. Marciniak ${ }^{1}$, T. Michałowski ${ }^{1}$, M. Kaasalainen ${ }^{2}$, J. Ďurech ${ }^{3}$, M. Polińska ${ }^{1}$, T. Kwiatkowski ${ }^{1}$, A. Kryszczyńska ${ }^{1}$, \\ R. Hirsch ${ }^{1}$, K. Kamiński ${ }^{1}$, M. Fagas ${ }^{1}$, F. Colas ${ }^{4}$, S. Fauvaud ${ }^{5,6}$, G. Santacana ${ }^{5}$, R. Behrend ${ }^{7}$, and R. Roy ${ }^{8}$ \\ 1 Astronomical Observatory, Adam Mickiewicz University, Słoneczna 36, 60-286 Poznań, Poland \\ e-mail: aniab@lab.astro.amu.edu.pl \\ Rolf Nevanlinna Institute, University of Helsinki PO Box 4, 00014 Helsinki, Finland \\ Astronomical Institute, Charles University in Prague V Holešovičkách 2, 18000 Prague, Czech Republic \\ Institut de Mecanique Celeste, 77 Av. Denfert Rochereau, 75014 Paris, France \\ Association AstroQueyras, Le Bois de Bardon, 16110 Taponnat, France \\ ${ }^{6}$ Groupe Européen d'Observations Stellaires (GEOS), 23 Parc de Levesville, 28300 Bailleau-1'Evêque, France \\ 7 Geneva Observatory, 1290 Sauverny, Switzerland \\ 8 Observatoire de Blauvac, 84570 St-Estève, France
}

Received 23 April 2007 / Accepted 15 June 2007

\section{ABSTRACT}

\begin{abstract}
We report photometric lightcurve observations of 184 Dejopeja (apparition years: 2000, 2002, 2005, 2006), 276 Adelheid $(2000,2001,2004,2005,2006)$, and 556 Phyllis $(1998,2000,2002,2004,2005,2006)$ carried out on 48 nights at four observatories. Using all of the available lightcurves, the spin vectors, senses of rotation, and shape models of these three asteroids have been determined.
\end{abstract}

Key words. techniques: photometric - minor planets, asteroids

\section{Introduction}

Modelling of the physical parameters of an asteroid on the basis of brightness measurements requires observations at a few apparitions at the largest possible span of longitudes and phase angles. So far, the parameters of almost 200 asteroids have been modelled, such as the rotational spin axes, sidereal periods, senses of rotation, and even their three-dimensional shapes. The last was possible using the convex inversion method.

Knowledge of the asteroid parameters is essential for understanding the formation and further evolution of the Solar System. To investigate these issues, one needs the largest possible sample of the asteroid spin vectors. Then, interesting facts are revealed, like the grouping of asteroid spin axes in the Koronis family (Slivan 2002) or a large excess of the near - Earth asteroids rotating in a retrograde sense (La Spina et al. 2004).

The lightcurves of over 2000 asteroids have been collected in the photometric database, but most of these objects were observed once or twice, which is not enough for proper modelling. But adding data from two or more new apparitions to the existing set would improve the situation a lot. It is worth stressing that only repeated observations of the same asteroids give the desired results.

This paper, fourth in the series, gives models of three new asteroids, compiling new observations with the previously published ones. These models and future ones will be included in a still growing database of spin parameters at http://www.astro.amu.edu.pl/Science/Asteroids and a database of the asteroid shape models at http://astro. troja.mff.cuni.cz/projects/asteroids3D

\section{Observations of three main belt asteroids}

Photometric observations of 184 Dejopeja, 276 Adelheid, and 556 Phyllis on 48 nights in the years 1998-2006 were made at four observatories. A large majority of the data came from the Borowiec Station of Poznań Astronomical Observatory (Poland). Other observatories were: Pic de Château-Renard Observatory, Pic du Midi Observatory, and Blauvac Observatory, all situated in France.

The Borowiec Observatory is equipped with a $0.4-\mathrm{m}, \mathrm{F} / 4.5$ Newton reflector, a KAF400 CCD camera, and a set of Bessel BVRI filters. A clear-glass filter was used in all of these observations, so the observations were not transformed to the standard system; see Michałowski et al. (2004) for a full description of the instrument and the reduction procedure.

Observations of other asteroids performed in Borowiec have been already published, together with the new models in, for example, Michałowski et al. (2000, 2001), Kryszczy ńska et al. (2003), Durech et al. (2007), and in the three previous papers of the present series - Michałowski et al. (2004, 2005, 2006).

At Pic de Château-Renard, a 0.62-m, F/3 Cassegrain telescope with a KAF400 CCD camera was used. Asteroids were observed through the filters $R$ and $V$. The photometry was relative, as in all presented cases. A $1.05-\mathrm{m}$ telescope with a Thomson 7863 CCD camera and an $R$ filter were used at the Pic du Midi Observatory, while the Blauvac Observatory is equipped with a $0.31-\mathrm{m}$ telescope.

Table 1 contains the aspect data for the three observed asteroids. In the first column there is the date of observation referring to the mid-time of the observed lightcurve. The subsequent columns are the distances (in AU) from the asteroid to the Sun 
Table 1. Aspect data.

\begin{tabular}{|c|c|c|c|c|c|c|}
\hline \multirow[t]{2}{*}{ Date (UT) } & \multirow{2}{*}{$\begin{array}{c}r \\
(\mathrm{AU}) \\
\end{array}$} & \multirow{2}{*}{$\begin{array}{l}\Delta \\
(\mathrm{AU}) \\
\end{array}$} & \multirow{2}{*}{$\begin{array}{c}\text { Phase } \\
\text { angle } \\
\left({ }^{\circ}\right) \\
\end{array}$} & \multicolumn{2}{|c|}{$\bar{l}_{(\mathrm{J} 2000)} \beta$} & \multirow[t]{2}{*}{ Obs. } \\
\hline & & & & $\left(^{\circ}\right)$ & $\left({ }^{\circ}\right)$ & \\
\hline \multicolumn{7}{|l|}{184 Dejopeja } \\
\hline 20000923.1 & 3.427 & 2.465 & 5.64 & 19.95 & 1.06 & Bor \\
\hline 20000924.0 & 3.426 & 2.461 & 5.36 & 19.80 & 1.06 & Bor \\
\hline 20020202.8 & 3.150 & 2.475 & 14.78 & 79.14 & 1.25 & Bor \\
\hline 20020203.9 & 3.149 & 2.485 & 14.97 & 79.12 & 1.24 & Bor \\
\hline 20050914.0 & 3.398 & 2.444 & 6.32 & 329.38 & 0.09 & Bla \\
\hline 20050922.9 & 3.401 & 2.507 & 8.96 & 328.10 & 0.12 & Bla \\
\hline 20050929.9 & 3.403 & 2.570 & 10.79 & 327.33 & 0.15 & Bla \\
\hline 20051010.8 & 3.406 & 2.688 & 13.16 & 326.61 & 0.18 & Bor \\
\hline 20051011.8 & 3.406 & 2.700 & 13.35 & 326.57 & 0.18 & Bor \\
\hline 20051015.8 & 3.407 & 2.748 & 14.04 & 326.48 & 0.20 & Bor \\
\hline 2006099.0 & 3.388 & 2.750 & 14.67 & 44.56 & 1.19 & Bor \\
\hline 20060915.0 & 3.386 & 2.677 & 13.62 & 44.43 & 1.23 & Bor \\
\hline 20060926.1 & 3.382 & 2.559 & 11.20 & 43.70 & 1.3 & Bor \\
\hline 20061001.0 & 3.380 & 2.514 & 9.91 & 43.17 & 1.34 & Bor \\
\hline 20061017.0 & 3.373 & 2.409 & 5.06 & 40.76 & 1.43 & Bor \\
\hline 20061025.9 & 3.370 & 2.380 & 2.05 & 39.09 & 1.47 & Bor \\
\hline \multicolumn{7}{|l|}{276 Adelheid } \\
\hline 20000826.0 & 3.248 & 2.366 & 10.23 & 2.75 & 18.95 & Bor \\
\hline 20000826.9 & 3.247 & 2.359 & 10.01 & 2.61 & 18.94 & Bor \\
\hline 20000827.9 & 3.247 & 2.352 & 9.77 & 2.45 & 18.93 & Bor \\
\hline 20000921.9 & 3.232 & 2.262 & 5.47 & 357.42 & 17.80 & Bor \\
\hline 20011026.1 & 2.965 & 2.234 & 15.06 & 81.22 & -17.05 & ChR \\
\hline 20011028.0 & 2.964 & 2.214 & 14.63 & 81.10 & -17.37 & ChR \\
\hline 20040514.0 & 3.244 & 2.305 & 7.86 & 252.41 & 18.25 & Bor \\
\hline 20040515.0 & 3.245 & 2.302 & 7.66 & 252.22 & 18.35 & Bor \\
\hline 20040528.9 & 3.252 & 2.280 & 6.01 & 249.35 & 19.58 & Bor \\
\hline 20040530.0 & 3.253 & 2.280 & 6.02 & 249.13 & 19.65 & Bor \\
\hline 20050917.9 & 3.313 & 2.532 & 12.53 & 316.07 & 25.99 & Bor \\
\hline 20050918.9 & 3.312 & 2.540 & 12.70 & 315.97 & 25.88 & Bor \\
\hline 20050922.9 & 3.311 & 2.573 & 13.37 & 315.60 & 25.36 & Bor \\
\hline 20050926.8 & 3.310 & 2.609 & 14.00 & 315.33 & 24.84 & Bor \\
\hline 20051004.9 & 3.307 & 2.689 & 15.14 & 315.05 & 23.74 & Bor \\
\hline 20060919.0 & 3.107 & 2.314 & 13.22 & 40.88 & 1.99 & Bor \\
\hline 20060927.1 & 3.101 & 2.235 & 11.01 & 40.02 & 1.31 & Bor \\
\hline 20061018.0 & 3.086 & 2.104 & 3.81 & 36.35 & -0.67 & Bor \\
\hline \multicolumn{7}{|l|}{556 Phyllis } \\
\hline 19980130.1 & 2.2319 & 1.2489 & 2.14 & 126.56 & -3.48 & ChR \\
\hline 19980130.9 & 2.2323 & 1.2498 & 2.48 & 126.36 & -3.51 & ChR \\
\hline 20000827.0 & 2.5362 & 1.6098 & 11.46 & 3.07 & 7.52 & Bor \\
\hline 20000831.0 & 2.5320 & 1.5838 & 9.92 & 2.39 & 7.69 & Bor \\
\hline 20020108.1 & 2.2454 & 1.4590 & 18.78 & 154.82 & -4.01 & ChR \\
\hline 20020110.2 & 2.2466 & 1.4418 & 18.08 & 154.74 & -4.14 & ChR \\
\hline 20020129.2 & 2.2592 & 1.3232 & 10.18 & 152.40 & -5.28 & Pic \\
\hline 20020201.2 & 2.2614 & 1.3114 & 8.72 & 151.79 & -5.45 & Pic \\
\hline 20020309.0 & 2.2900 & 1.3538 & 10.75 & 143.53 & -6.56 & Bor \\
\hline 20040905.1 & 2.4736 & 1.6010 & 14.58 & 20.27 & 7.96 & Bor \\
\hline 20040913.9 & 2.4638 & 1.5343 & 11.36 & 19.08 & 8.33 & Bor \\
\hline 20040916.9 & 2.4605 & 1.5153 & 10.17 & 18.56 & 8.44 & Bor \\
\hline 20040918.1 & 2.4591 & 1.5083 & 9.68 & 18.34 & 8.48 & Bor \\
\hline 20051230.2 & 2.2733 & 1.7923 & 24.54 & 172.23 & -4.39 & Bor \\
\hline 20060110.1 & 2.2821 & 1.6738 & 22.81 & 173.75 & -5.01 & Bor \\
\hline 20060116.2 & 2.2871 & 1.6135 & 21.52 & 174.21 & -5.38 & Bor \\
\hline
\end{tabular}

Observatory Code: Bor - Borowiec; Bla - Blauvac; ChR - Chateau-Renard; Pic - Pic du Midi.

and the Earth, the solar phase angle, the $J 2000.0$ ecliptic longitude $(\lambda)$, and latitude $(\beta)$, given for the time from the first column. The codes of the observatories are listed in the last column of the table.

The basic physical parameters of the asteroids are given in Table 2. Their IRAS diameters $(D)$, albedoes, and taxonomic types are taken from The Small Bodies Node of the NASA Planetary Data System (http: //pdssbn . astro.umd.edu/).
Table 2. Asteroid parameters.

\begin{tabular}{lcrc}
\hline \hline Asteroid & $D(\mathrm{~km})$ & albedo & Type \\
\hline 184 Dejopeja & 66 & 0.190 & $\mathrm{X}$ \\
276 Adelheid & 122 & 0.045 & $\mathrm{X}$ \\
556 Phyllis & 38 & 0.185 & $\mathrm{~S}$ \\
\hline
\end{tabular}




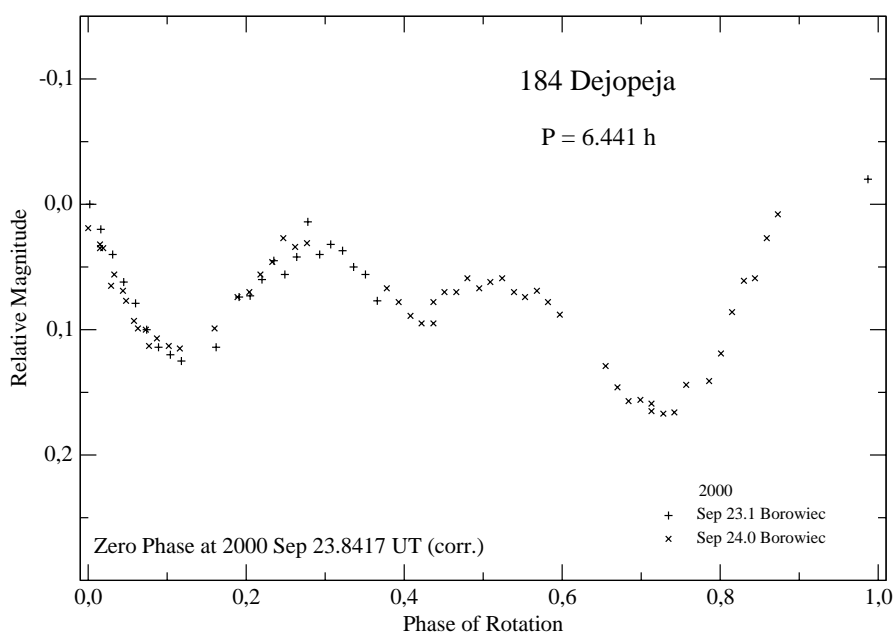

Fig. 1. Lightcurve of 184 Dejopeja in 2000.

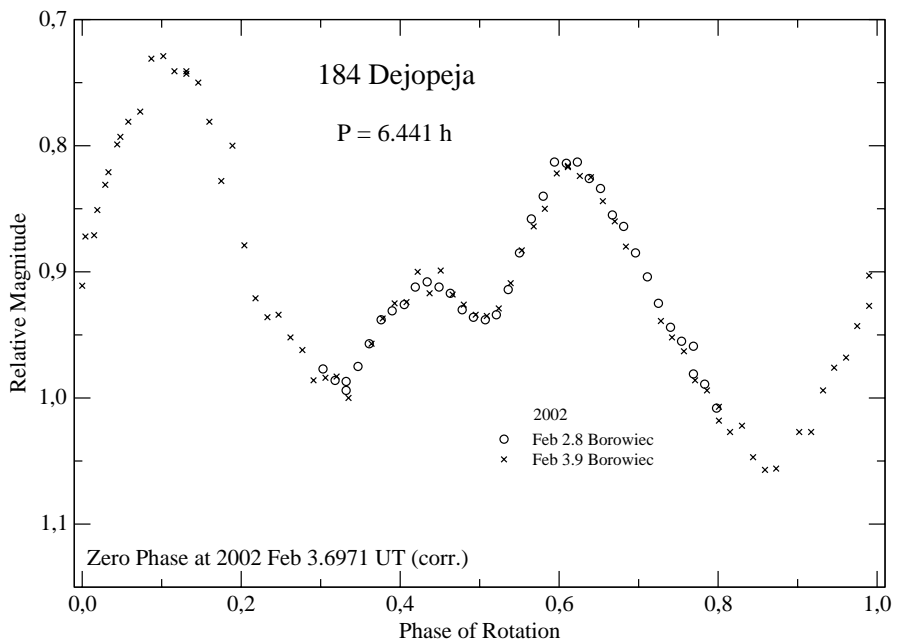

Fig. 2. Lightcurve of 184 Dejopeja in 2002.

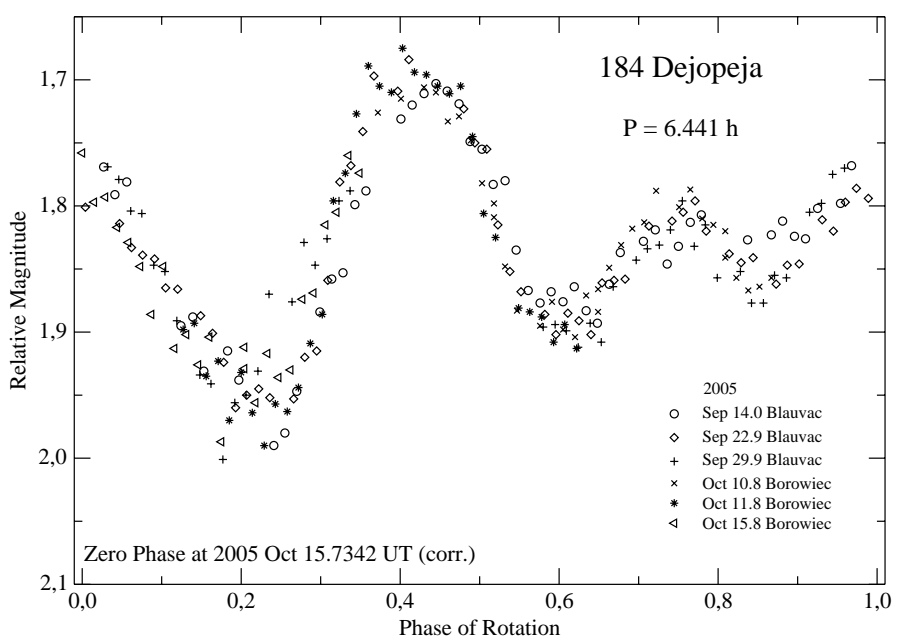

Fig. 3. Lightcurve of 184 Dejopeja in 2005.

Results of our new observations are presented in Figs. 1-14 in the form of composite lightcurves. They were created using the procedure described by Magnusson \& Lagerkvist (1990). The lightcurves from individual oppositions were composited with the synodical periods written in the graphs. The points from different nights are marked with different symbols. The vertical

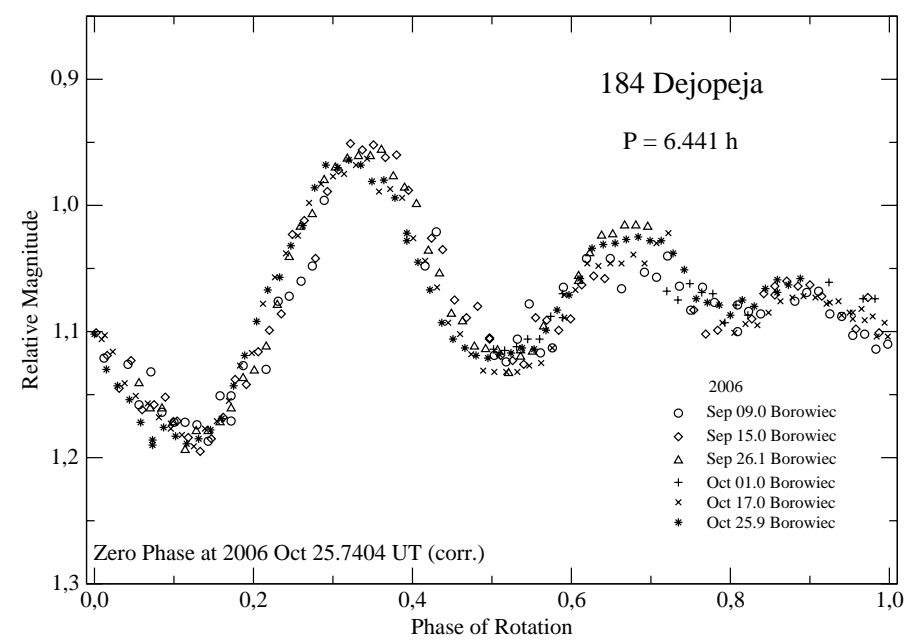

Fig. 4. Lightcurve of 184 Dejopeja in 2006.

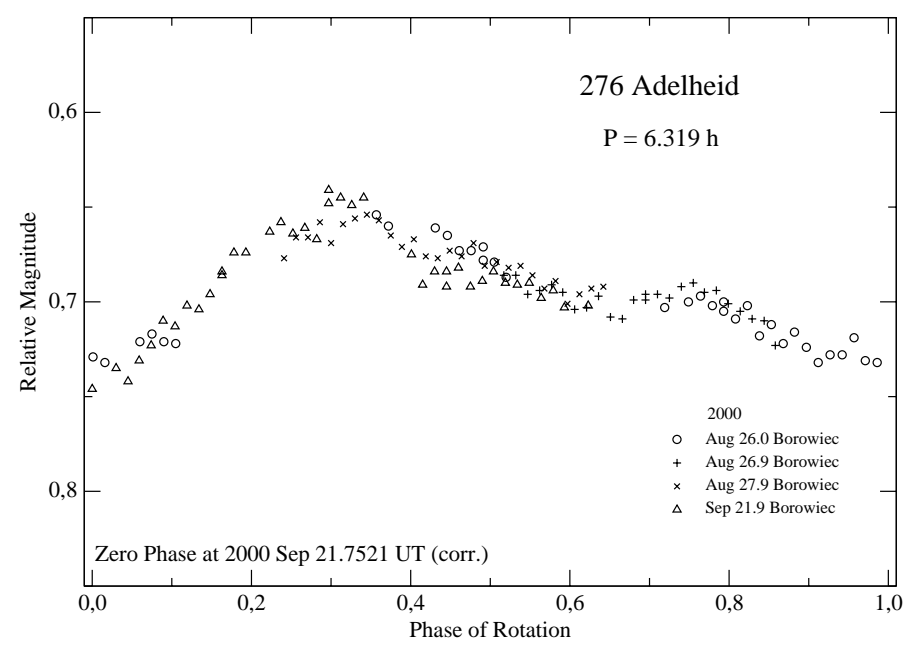

Fig. 5. Lightcurve of 276 Adelheid in 2000.

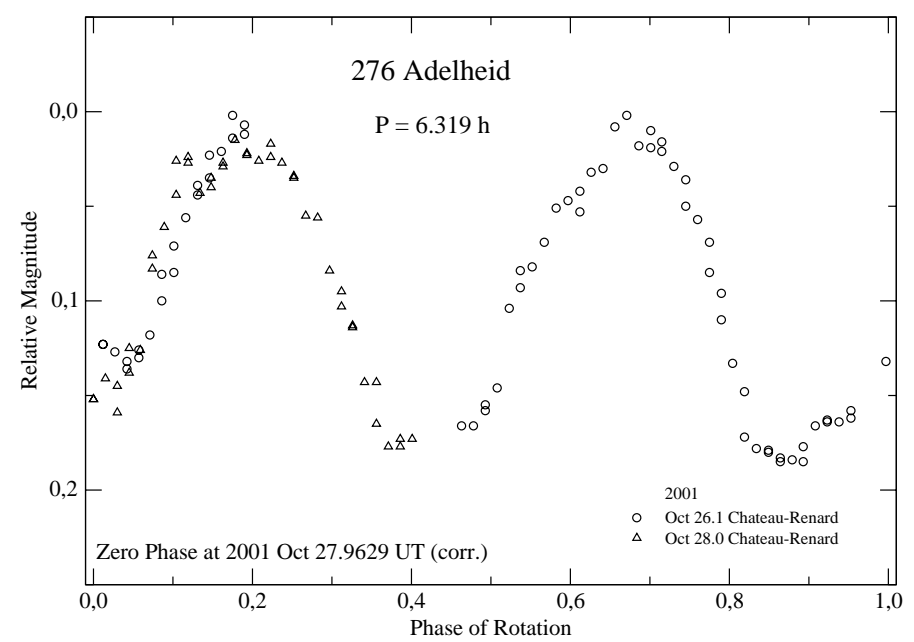

Fig. 6. Lightcurve of 276 Adelheid in 2001.

position of each individual lightcurve is obtained to minimize the dispersion of data points relative to their neighbours. The abscissae are the rotational phases with the zero points corrected for light-time. 


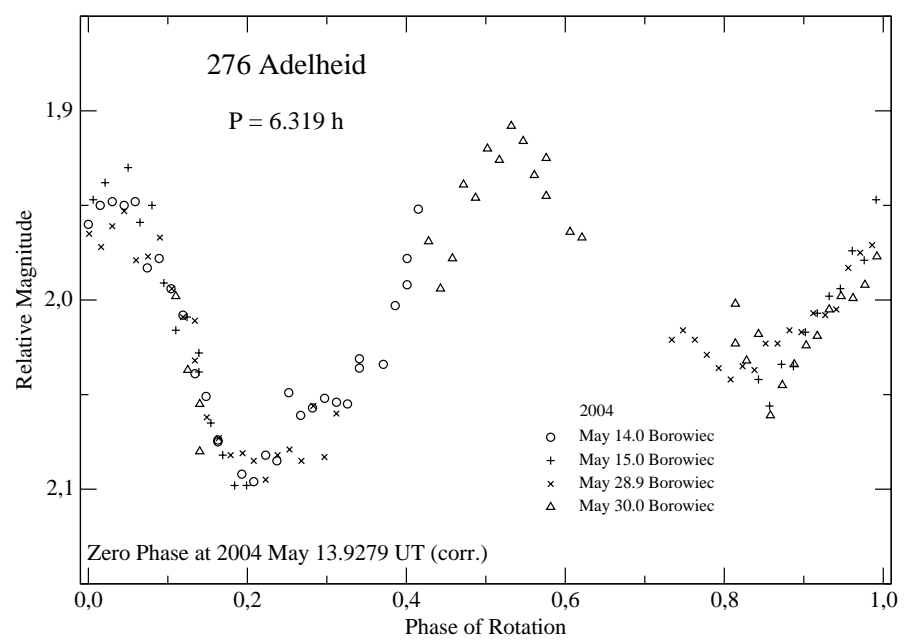

Fig. 7. Lightcurve of 276 Adelheid in 2004.

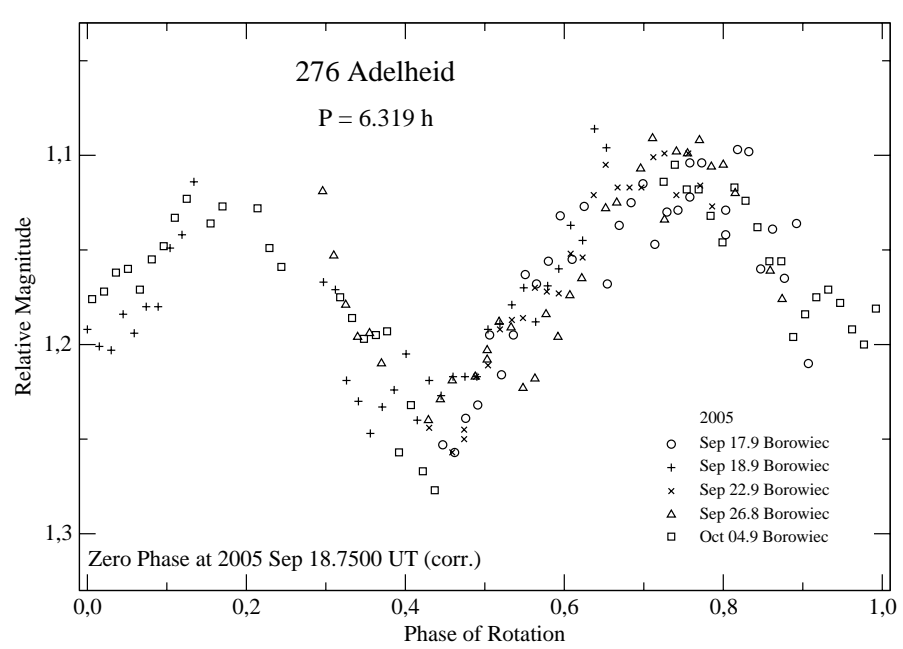

Fig. 8. Lightcurve of 276 Adelheid in 2005.

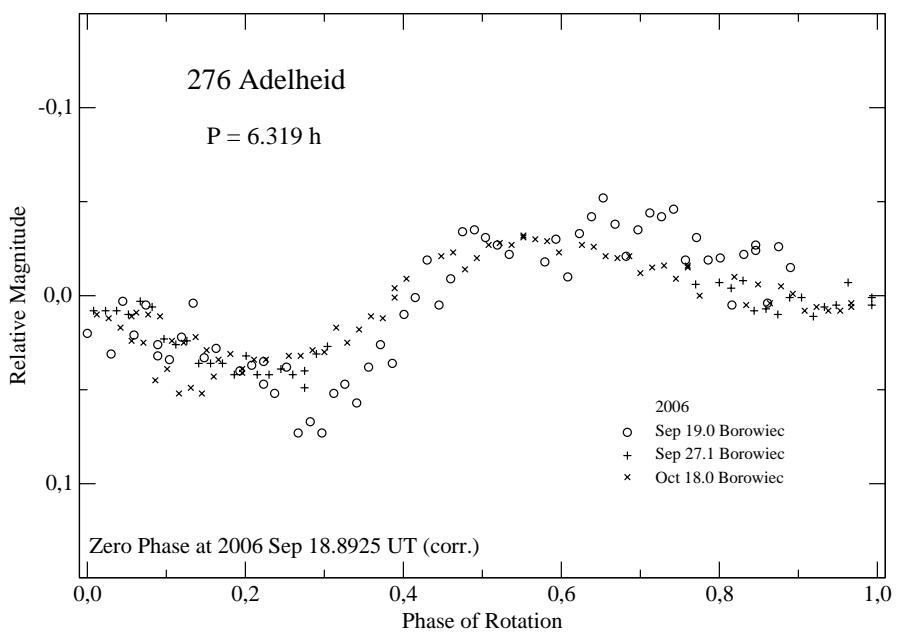

Fig. 9. Lightcurve of 276 Adelheid in 2006.

\subsection{Dejopeja}

Dejopeja was first observed by Tedesco (1979). The tri-modality of its lightcurve was already visible at that time. It was only a one-night run on 9 September 1977, with the estimated period of $6.7 \mathrm{~h}$, and the amplitude about $0.21 \mathrm{mag}$. The next photometric observations of this asteroid were made by Gil-Hutton (1995).

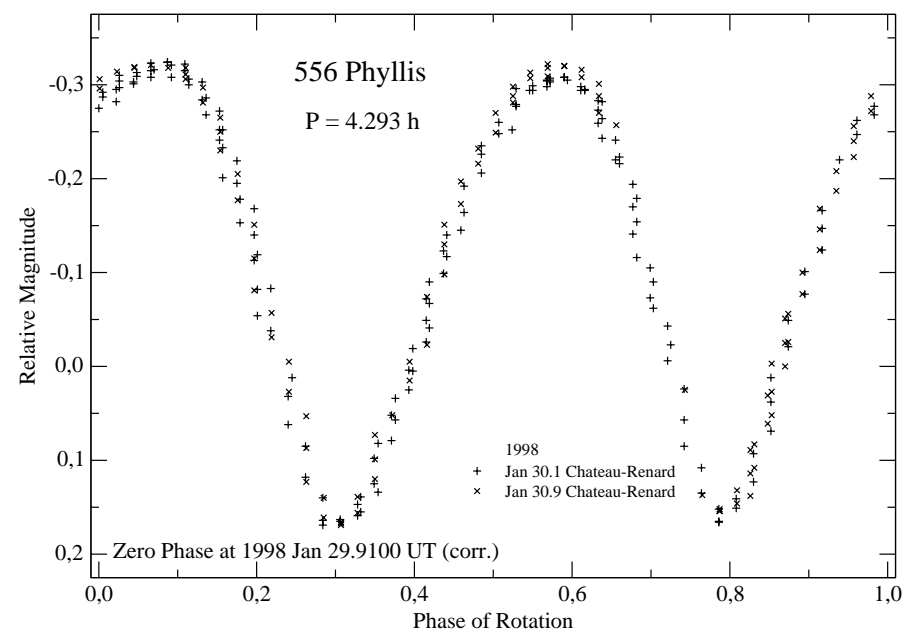

Fig. 10. Lightcurve of 556 Phyllis in 1998.

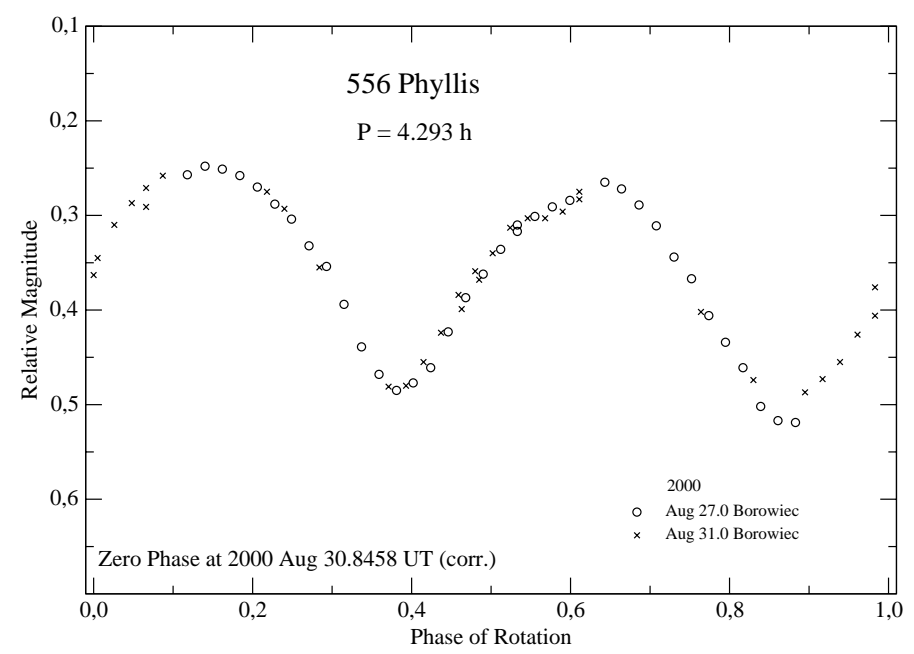

Fig. 11. Lightcurve of 556 Phyllis in 2000.

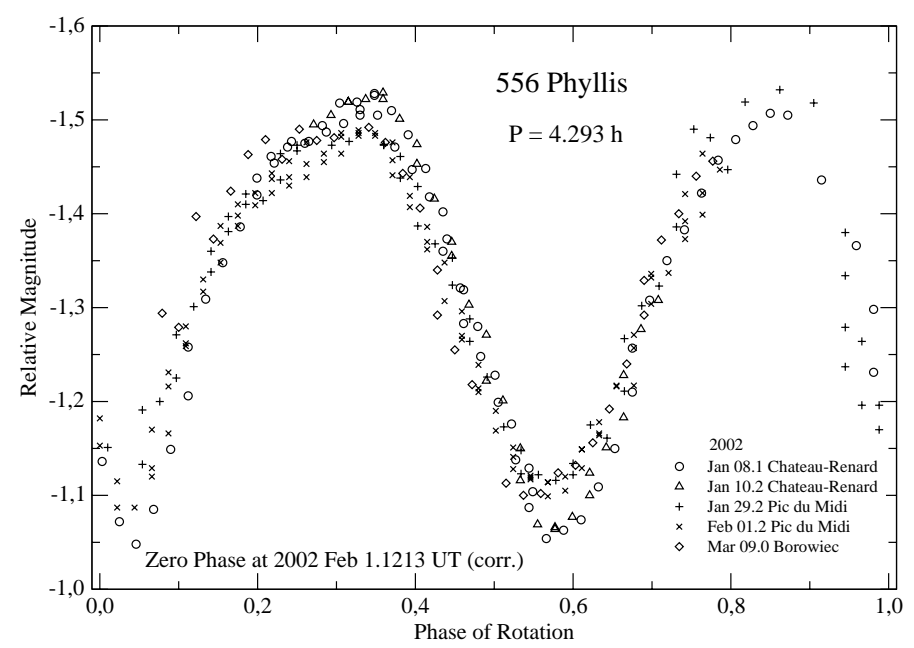

Fig. 12. Lightcurve of 556 Phyllis in 2002.

Observed during three nights in April 1992, Dejopeja showed unequal extrema with an amplitude over $0.28 \mathrm{mag}$. The period was determined as $6.455 \pm 0.008 \mathrm{~h}$, but the composite lightcurve had gaps.

We observed 184 Dejopeja on 13 nights during four apparitions in the years 2000, 2002, 2005, and 2006. The composite 


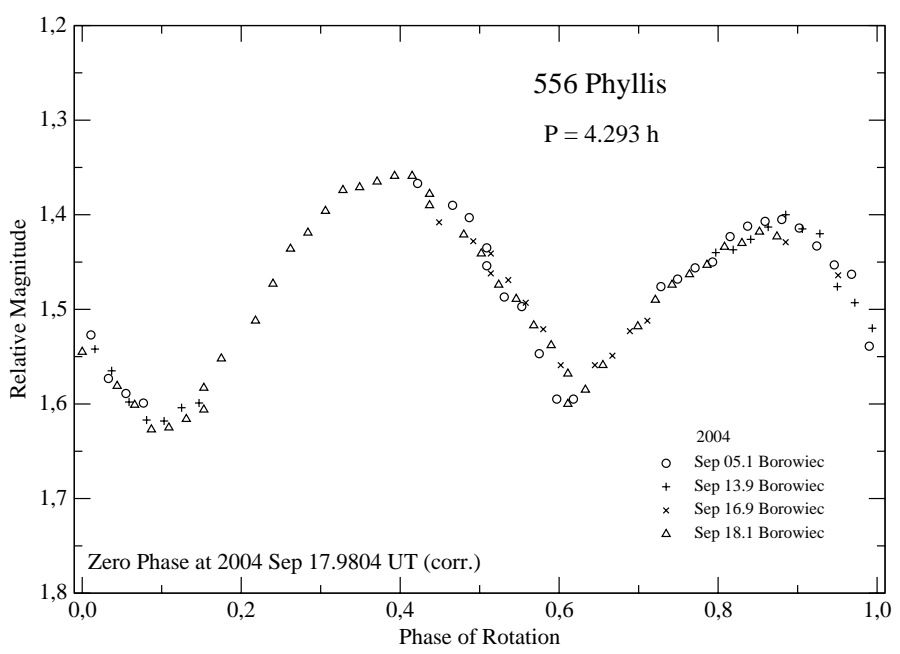

Fig. 13. Lightcurve of 556 Phyllis in 2004.

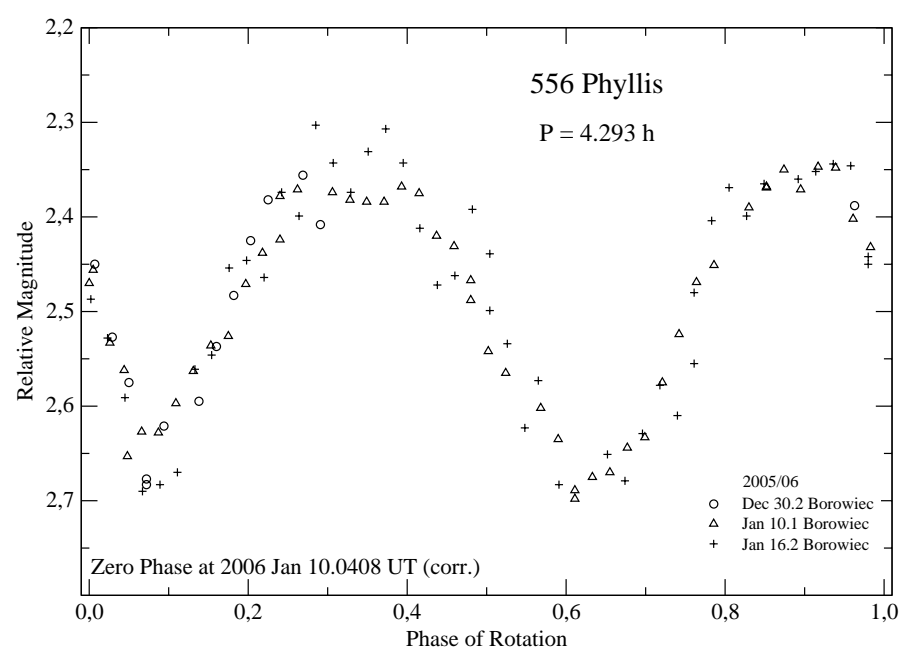

Fig. 14. Lightcurve of 556 Phyllis in 2006.

lightcurves are constructed with the synodical period of $6.441 \pm$ $0.001 \mathrm{~h}$, which is a little shorter than the value given by GilHutton (1995).

The lightcurve from two nights in September 2000 (Fig. 1) was apparently tri-modal, but the third maximum was lacking due to the gap in observations. The coverage was almost $90 \%$ of the rotational cycle. The peak-to-peak amplitude was $0.19 \pm$ $0.01 \mathrm{mag}$, but it must have been larger at that time. On the fully covered lightcurve, from two nights in February 2002 (Fig. 2), there were three distinct maxima, each a different height. The amplitude was larger at $0.32 \pm 0.01 \mathrm{mag}$, and the estimated period was confirmed. On six nights in September-October 2005, we obtained a similar lightcurve (Fig. 3), but with more noise and with an amplitude of $0.25 \pm 0.03 \mathrm{mag}$. The "noise" of the composite lightcurve might be due to the changing phase angle over a month-long time span. In the 2006 apparition (Fig. 4), Dejopeja showed a lower amplitude of $0.22 \pm 0.01 \mathrm{mag}$ and a smaller difference between the two of the minima. The fullycovered lightcurve came from the observations on six nights in September-October 2006.

\subsection{Adelheid}

The first photometric observations of Adelheid were made by Carlsson \& Lagerkvist (1983). No lightcurve was given, but the colour indices were determined as $B-V=0.73$ and $U-B=$ 0.22 . Chronologically, the first lightcurve observations of this asteroid were conducted by Piironen et al. (1994), who observed it during eight nights between October and November 1984. The amplitude was very small, around $0.1 \mathrm{mag}$, but in the composite lightcurve there were two clearly visible pairs of extrema. The period determined was $6.32 \pm 0.02 \mathrm{~h}$.

The period determination was made by Dotto et al. (1994): $6.328 \pm 0.012 \mathrm{~h}$. DiMartino et al. (1995) published the observations from three nights between February and March 1992 made at ESO. The composite, bimodal lightcurve still had a small amplitude of 0.1 mag and was constructed with the period given by Dotto et al. (1994).

In November 2000, Wang \& Shi (2002) got two lightcurves of this object, unfortunately quite noisy; still, the change in the lightcurve was clearly visible. This time there was only one maximum and one minimum. The amplitude reached 0.18 mag and the period was $6.29 \pm 0.01 \mathrm{~h}$, close to the previous determinations. Pray (2005) observed Adelheid on four nights in June 2004. The second pair of extrema were again visible in that asymmetric lightcurve. The light variation was within $0.17 \mathrm{mag}$, with the period of $6.315 \pm 0.002 \mathrm{~h}$. Lastly, Adelheid was observed by Sada (2006). Five nights run in August 2005 confirmed the period and amplitude: $P=6.315 \pm 0.005 \mathrm{~h}, A=0.17 \mathrm{mag}$. All of the reported periods closely resemble each other.

We gathered the observational data of Adelheid on 19 nights in the years 2000, 2001, 2004, 2005, and 2006. The obtained synodical period is $6.319 \pm 0.001 \mathrm{~h}$, very close to the values from Pray and Sada.

On four nights in August and September 2000 (Fig. 5), the lightcurve of Adelheid showed one pair of extrema, with an indication of a second pair. The amplitude was small at $0.08 \pm$ 0.01 mag. The lightcurve from the next apparition (Fig. 6) was completely different: on two nights, in October 2001 Adelheid showed two extrema at the same levels, but with larger amplitude $0.17 \pm 0.01 \mathrm{mag}$ and irregurally shaped minima. In 2004 the situation changed again (Fig. 7). There were two extrema visible on four nights in May, but the lightcurve became more irregular, and the maximum amplitude was $0.17 \pm 0.02 \mathrm{mag}$. On five nights in September-October 2005 (Fig. 8) the amplitude of Adelheid lightcurves appeared to decrease to $0.13 \pm 0.03$ mag but the assymetry remained. Finally in the 2006 apparition (Fig. 9), the lightcurve switched to a single mode again. During the three nights observations in September-October 2006, the amplitude fell to $0.09 \pm 0.03$ mag.

\subsection{Phyllis}

On 16 November 1981 Zappala et al. (1983) observed this object, and constructed a clumpy lightcurve with a period of $4.28 \pm 0.002 \mathrm{~h}$, and $0.24 \mathrm{mag}$ amplitude. In November-December 1981 Harris et al. (1992) made three sparse lightcurve observations, from which they were unable to determine an unambiguous synodic rotational period. But adding the lightcurve by Zappala et al., they obtained a period of $4.2932 \pm 0.0004 \mathrm{~h}$. Such good precision was possible because the short rotation period of this asteroid resulted in many revolutions over the two-week observation span.

We observed the asteroid Phyllis during its five apparitions in 1998, 2000, 2002, 2004, and 2005-2006. The regular, bimodal lightcurves were composited with the synodical period of $4.293 \pm 0.001 \mathrm{~h}$, which was consistent with previous determinations. 
Table 3. Spin and shape models.

\begin{tabular}{|c|c|c|c|c|c|c|c|c|c|c|}
\hline \multirow{2}{*}{$\begin{array}{r}\text { Sidereal } \\
\text { period (hours) } \\
\end{array}$} & \multirow{2}{*}{$\begin{array}{c}\begin{array}{c}\text { Sense of } \\
\text { rotation }\end{array} \\
\end{array}$} & \multicolumn{2}{|c|}{ Pole 1} & \multicolumn{2}{|c|}{ Pole 2} & \multirow{2}{*}{$\begin{array}{c}\text { Observing span } \\
\text { (years) }\end{array}$} & \multirow[t]{2}{*}{$N_{\text {opp }}$} & \multirow[t]{2}{*}{ 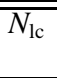 } & \multirow[t]{2}{*}{ Method } & \multirow[t]{2}{*}{ Reference } \\
\hline & & $\lambda_{p}$ & $\beta_{p}$ & $\lambda_{p}$ & $\beta_{p}$ & & & & & \\
\hline $\begin{array}{r}184 \text { Dejopeja } \\
6.441111\end{array}$ & $\mathrm{P}$ & $18^{\circ}$ & $+54^{\circ}$ & $201^{\circ}$ & $+52^{\circ}$ & $1977-2006$ & 6 & 17 & $\mathrm{~L}$ & Present work \\
\hline $\begin{array}{r}276 \text { Adelheid } \\
6.319204\end{array}$ & $\mathrm{R}$ & $9^{\circ}$ & $-4^{\circ}$ & $198^{\circ}$ & $-20^{\circ}$ & 1984-2006 & 7 & 31 & $\mathrm{~L}$ & Present work \\
\hline $\begin{array}{r}556 \text { Phyllis } \\
4.292623\end{array}$ & $\mathrm{P}$ & $35^{\circ}$ & $+55^{\circ}$ & $209^{\circ}$ & $+41^{\circ}$ & 1981-2006 & 6 & 19 & $\mathrm{~L}$ & Present work \\
\hline
\end{tabular}

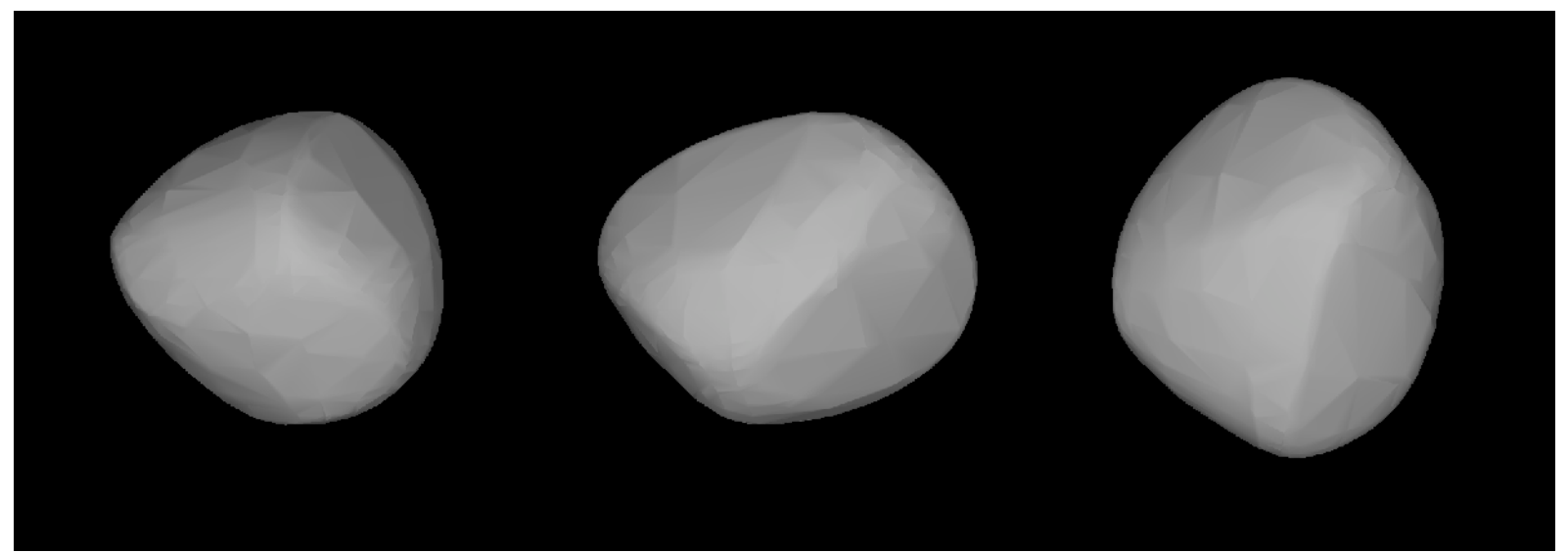

Fig. 15. Shape model of 184 Dejopeja, shown at equatorial viewing and illumination geometry, with rotational phases $90^{\circ}$ apart (two pictures in the left) and the pole-on view to the right.

On two nights in January 1998, Phyllis showed a regular lightcurve with a large amplitude of $0.48 \pm 0.01$ mag (Fig. 10). There was a slight asymmetry in the shape of the extrema. In the apparition in August 2000, the two-night lightcurve showed a much smaller amplitude of $0.27 \pm 0.01 \mathrm{mag}$, and some irregularity was still visible, especially in the second maximum (Fig. 11). We obtained the next lightcurve in January - March 2002 (Fig. 12). The changing amplitude resulted from the changing phase angle over the two-month observing span. The characteristic shape of the extrema was still visible, and the ampitude grew to $0.45 \pm 0.01 \mathrm{mag}$. On four nights in September 2004, the amplitude was $0.26 \pm 0.01 \mathrm{mag}$ and one minimum became quite sharp (Fig. 13). In the last apparition (Fig. 14) at the turn of the year 2005 and 2006, our three-night noisy lightcurve had $0.33 \pm 0.03$ mag amplitude.

\section{Pole and shape results of the asteroids observed}

The pole and period solution, together with a three-dimensional convex shape model, were obtained using the lightcurve inversion method by Mikko Kaasalainen (Kaasalainen \& Torppa 2001; and Kaasalainen et al. 2001, 2003). The method uses all the available data points and models the shape, along with spin and scattering properties, using a large number of parameters. The photometric data can be absolute and/or relative. The model lightcurves closely fit the observed ones, within the noise level. As in the other methods, one often obtains two solutions, with a $\lambda_{p} \pm 180^{\circ}$ ambiguity for the asteroids moving close to the ecliptic plane.

Table 3 presents the results. The first column contains the sidereal period in hours, where the uncertainty is limited to the last digit, as the method determines the rotation period very well. Next, there is the sense of rotation $(P-$ prograde and
$R$ - retrograde) and two pole solutions. They are given as the ecliptic coordinates of the north asteroids' poles for a J2000 epoch. Here, the error is usually at the level of $\pm 5^{\circ}$ on the celestial sphere and is influenced by the systematic errors present in lightcurves and model errors rather than the formal error derived from the observational noise level. The errors given here were estimated based on comparing of various models obtained from lightcurve inversion with different starting parameters. The resulting pole positions always fell within $\pm 5^{\circ}$ of each other and the period remained the same, varying only on the last digit. More details on error estimation in the lightcurve inversion method are given in Torppa et al. (2003), and Kaasalainen \& Ďurech (2007)

Table 3 also shows the observing span in years, the number of oppositions, and the number of all lightcurves used for modelling. This table finally gives the method used (L stands for lightcurve inversion) and the reference. There are no previously published pole results for these three asteroids.

We constructed the model of 184 Dejopeja using 17 lightcurves from 6 apparitions (1977, 1992, 2000, 2002, 2005, and 2006). But even before we got all the data, we were able to obtain a uniqe model, with only 11 lightcurves from 5 apparitions. That was probably due to the atypical tri-modal lightcurve of this asteroid caused by its angular shape. Usually, many more lightcurves are needed. The obtained prograde sense of rotation and two pole solutions are indicated in Table 3. The shape model of Dejopeja is shown in Fig. 15.

The data from seven apparitions are available for the asteroid 276 Adelheid (1984, 1992, 2000, 2001, 2004, 2005, and 2006). We used 31 lightcurves to obtain a model, after excluding especially noisy ones. Adelheid was found to be a retrograde rotator with an angular shape (Fig. 16) that made the second pair of the 


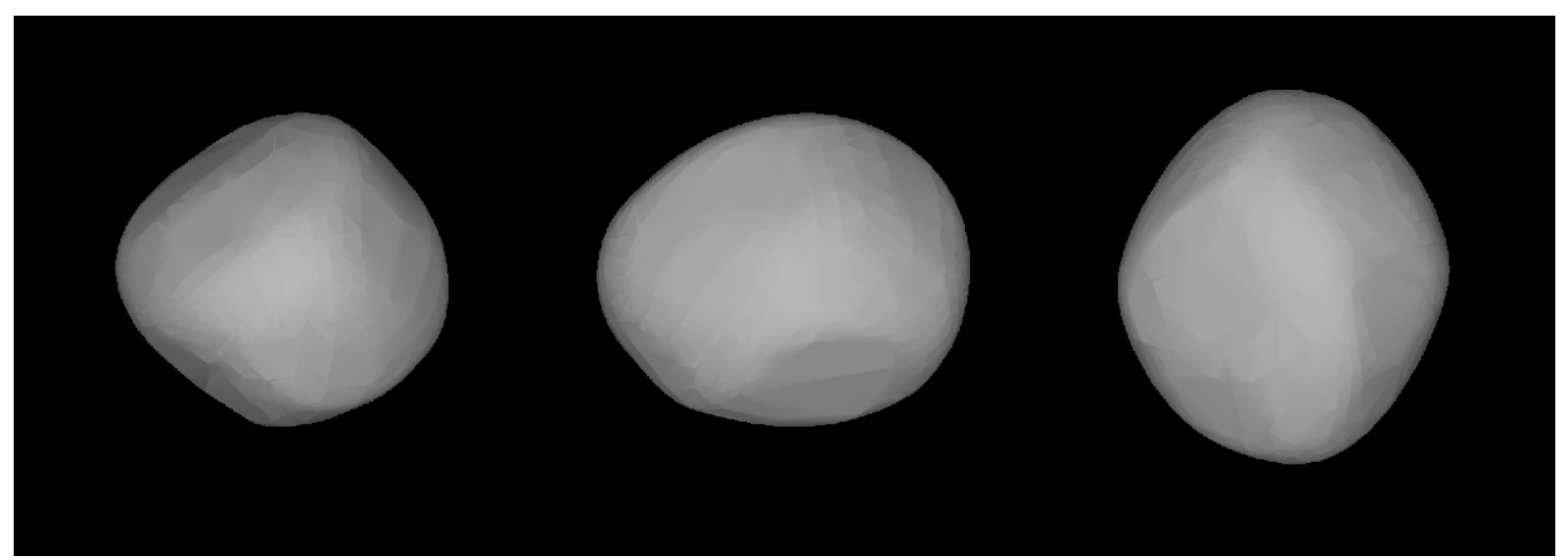

Fig. 16. Shape model of 276 Adelheid.

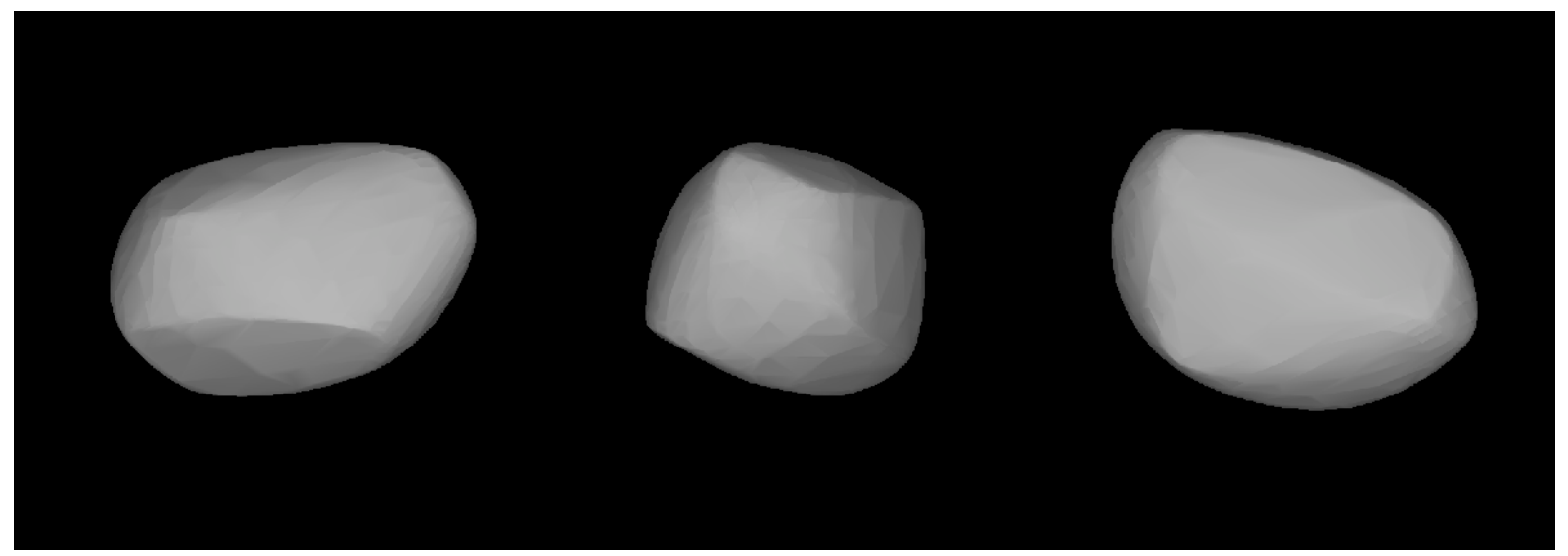

Fig. 17. Shape model of 556 Phyllis.

extrema switch on and off. There are two possible pole solutions (Table 3), one quite close to the ecliptic plane, which is rather rare.

Phyllis is the elongated asteroid producing regular lightcurves. Using all the 6 oppositions available (1981, 1998, 2000, 2002, 2004, and 2006) and 19 lightcurves, we obtained a model with the prograde sense of rotation and a pole at medium latitude (Table 3). Figure 17 shows the shape model of Phyllis.

Acknowledgements. Borowiec observations were reduced with the CCLRS STARLINK package.

This work was supported by the Polish Grant 1 P03D 02027.

The work of J.D. was supported by the Czech Grant Agency through grant GAČR 205/07/P070. M.K. is supported by the Academy of Finland. S.F. is grateful to F. Bererd, M. Fauvaud, N. Heck, D. Jolivet, A. Paschke, J.-P. Sareyan, and $\mathrm{H}$. Wucher for their help and technical assistance during observing runs.

\section{References}

Carlsson, M., \& Lagerkvist, C.-I. 1983, A\&AS, 53, 157

Di Martino, M., Dotto, E., Celino, A., Barucci, M. A., \& Fulchignoni, M. 1995, A\&AS, 112, 1

Dotto, E., Rotundi, A., \& De Sanctis, M. C. 1992, in Physical properties of small solar system bodies. ed. A. Brahic, J.-C. Gerard, \& J. Surdej, Université de Liège, Institut d'Astrophysique, Liège, 211
Durech, J., Kaasalainen, M., \& Marciniak, A. and 39 coauthors 2007, A\&A, 465, 331

Gil-Hutton, R. 1995, Rev. Mex. Astron. Astrofis., 31, 19

Harris, A. W., Young, J. W., Dockweiler, T., et al. 1992, Icarus, 95, 115

Kaasalainen, M., \& Torppa, J. 2001, Icarus, 153, 24

Kaasalainen, M., \& Durech, J. 2007, in Near Earth Objects, our Celestial Neighbors: Opportunity and Risk, ed. A. Milani, G. B. Valsecchi, \& D. Vokrouhlicky (Cambridge: Cambridge University Press), in press

Kaasalainen, M., Torrpa, J., \& Muinonen, K. 2001, Icarus, 153, 37

Kaasalainen, M., Mottola, S., \& Fulchignoni, M. 2003, in Asteroids III, ed. W. F. Bottke, A. Cellino, P. Paolicchi, \& R. P. Binzel (Univ. Arizona Press), 139 Kryszczyńska, A., Kwiatkowski, T., \& Michałowski, T. 2003, A\&A, 404, 729 La Spina, A., Paolicchi, P., Kryszczyńska, A., \& Pravec, P. 2004, Nature, 428, 400

Magnusson, P., \& Lagerkvist, C.-I. 1990, A\&AS, 86, 45

Michałowski, T., Pych, W., Berthier, J., et al. 2000, A\&AS, 146, 471

Michałowski, T., Pych, W., Kwiatkowski, T., et al. 2001, A\&A, 371, 748

Michałowski, T., Kwiatkowski, T., Kaasalainen, M., et al. 2004, A\&A, 416, 353 Michałowski, T., Kaasalainen, M., Marciniak, A., et al. 2005, A\&A, 443, 329 Michałowski, T., Kaasalainen, M., Polińska, M., et al. 2006, A\&A, 459, 663 Piironen, J., Bowell, E., Erikson, A., \& Magnusson, P. 1994, A\&AS, 106, 587 Pray, D. 2005, Minor Planet Bul., 32, 8

Sada, P. V. 2006, Minor Planet Bul., 33, 78

Slivan, S. M. 2002, Nature, 419, 49

Tedesco, E. F. 1979, Ph.D. Dissertation, New. Mex. State Univ., 280

Torppa, J., Kaasalainen, M., Michalowski, T., et al. 2003, Icarus, 164, 346 Wang, X.-B., \& Shi, Y. 2002, Earth, Moon and Planets, 91, 181

Zappala, V., Scaltriti, F., \& Di Martino, M. 1983, Icarus, 56, 325 